\title{
BMJ Global Health Remote evaluations of violence against women and girls interventions: a rapid scoping review of tools, ethics and safety
}

\author{
Ilana Seff, ${ }^{1}$ Luissa Vahedi, ${ }^{1}$ Samantha McNelly, ${ }^{1}$ Elfriede Kormawa, ${ }^{2}$ \\ Lindsay Stark (1) ${ }^{1}$
}

To cite: Seff I, Vahedi L, McNelly S, et al. Remote evaluations of violence against women and girls interventions: a rapid scoping review of tools, ethics and safety. BMJ Global Health 2021;6:e006780. doi:10.1136/ bmjgh-2021-006780

Handling editor Seye Abimbola

- Additional supplemental material is published online only. To view, please visit the journal online (http://dx.doi.org/10. 1136/bmjgh-2021-006780)

Received 1 July 2021 Accepted 19 August 2021

Check for updates

C Author(s) (or their employer(s)) 2021. Re-use permitted under CC BY-NC. No commercial re-use. See rights and permissions. Published by BMJ.

${ }^{1}$ Brown School of Social Work, Washington University in St Louis, St Louis, Missouri, USA ${ }^{2}$ UNICEF Nigeria, Abuja, Nigeria

Correspondence to

Dr Lindsay Stark:

lindsaystark@wustl.edu

\section{ABSTRACT}

Although programmes and policies targeting violence against women and girls (VAWG) have increased in the past decade, there is a paucity of evidence on the effectiveness of these interventions. To expand this evidence base, researchers increasingly employ remote data collection (RDC) - including online surveys, mobile applications and telephone interviews-in their evaluations. Although RDC allows for evaluations without in-person interactionswhich are restricted during crises such as the COVID-19 pandemic - information about these methods is necessary to understand their potential usefulness and limitations.

This scoping review examines remote evaluations of VAWG interventions to describe the landscape of RDC methods, reflect on safety and ethical considerations, and offer best practices for RDC in VAWG research. Fourteen studies met eligibility criteria, with seven, five, and two studies employing telephone interviews, online surveys, and mobile applications, respectively. Studies commonly stated that participants were asked to use a safe email or device, but the method for verifying such safety was rarely specified. Best practices around safety included creating a 'quick escape' button for online data collection to use when another individual was present, explaining to participants how to erase browsing history and application purchases, and asking participants to specify a safe time for researchers to call. Only eight studies established referral pathways for respondents as per best practice. None of the eligible studies took place in low/middle-income countries (LMICs) or humanitarian settings, likely reflecting the additional challenges to using RDC methods in lower resource settings. Findings were used to create a best practice checklist for programme evaluators and Institutional Review Boards using RDC for VAWG interventions. The authors found that opportunities exist for researchers to safely and effectively use RDC methodologies to gather VAWG data, but that further study is needed to gauge the feasibility of these methods in LMICs and humanitarian settings.

\section{INTRODUCTION}

Violence against women and girls (VAWG) continues to pose a significant threat to

\section{Key questions}

What is already known?

- Although the use of remote data collection (RDC) in evaluations of interventions targeting violence against women and girls (VAWG) has rapidly expanded during the COVID-19 pandemic, a systematic exploration of such methods has not been conducted.

- Collecting data on VAWG necessitates special considerations around safety and ethics, but more information is needed on how and to what extent these considerations are being incorporated into RDC methods.

What are the new findings?

- Telephone interviews have been the most commonly employed method of RDC for VAWG-related interventions, though the use of online surveys and mobile applications has increased in recent years.

- While safety and confidentiality protocols are limited, the responsibility of their maintenance is often placed on the study participants.

- To date, RDC methods have not been used to evaluate VAWG interventions in low/middle-income countries (LMICs) or humanitarian settings.

What do the new findings imply?

- RDC methods have the potential to be used safely and effectively in VAWG research, dependent on researchers embedding mechanisms to promote safety across multiple stages of the study design.

- By considering simple questions and prompts outlined in the best practice checklist, researchers can more effectively and safely use RDC methods in VAWG research.

- Additional research is necessary to understand best practice use of VAWG RDC in LMICs and humanitarian settings.

women's rights and public health across the globe; 1 in 3-or 852 million-women have experienced physical or sexual violence from an intimate partner or sexual violence from a non-partner in their lifetime. ${ }^{1}$ In an effort 
to improve the lives of women and girls and achieve the sustainable development goals around physical and sexual violence against women, recent years have ushered in a proliferation of interventions and policies to prevent VAWG. However, the evidence on what works to prevent and reduce VAWG remains thin, and greater prioritisation is needed for implementing robust evaluations of relevant programmes and policies. ${ }^{2-4}$

The recent COVID-19 pandemic, which has resulted in national lockdowns, economic shutdown, closure of nonessential services and movement restrictions, has complicated ongoing efforts to evaluate VAWG programmes. Traditional methods of data collection and formal reporting mechanisms for VAWG may be impeded during the pandemic due to travel bans, movement restrictions, increased potential transmission for participants and study teams. ${ }^{5}$ To mitigate these challenges, a growing number of researchers have turned to digital and remote technologies to collect data on VAWG during the pandemic. Remote data collection (RDC) involves the use of technology to facilitate data collection in a way that replaces in-person interaction between participants and enumerators, meaning data collection poses no additional risk for propagating COVID-19 community transmission. Examples of RDC tools include online surveys, telephone interviews and mobile applications. ${ }^{6-8}$

Collecting data on VAWG, whether in person or virtually, requires careful and thoughtful planning on the part of researchers in order to minimise harm and maximise benefits to respondents. ${ }^{9}$ For instance, breaches in confidentiality compromised privacy during disclosures of violence, and inadequate referral pathways for respondents following data collection can all place respondents at greater risk of violence. ${ }^{9}{ }^{10}$ The remote collection of such data may present additional ethical and methodological challenges. Data collected online, for example, may be more vulnerable to data breaches when proper precautions are not taken. Online data collection also may result in lower rates of participation and retention across the span of a study. ${ }^{11}$ Data collected through telephone surveys, in particular, add another layer of complexity. As stay-at-home orders during COVID-19 have increased the proximity of abusers to their victims, there is a corresponding risk that research-related disclosures of violence will be overheard, triggering violence. ${ }^{12} 13$ Further, researchers collecting data during the pandemic must navigate all of these issues alongside recognition of the heightened risk of violence for women that accompanies pandemic control policies more generally, ${ }^{14-17}$ and the potential reduced available services for referrals.

While the deployment of RDC tools has understandably and rapidly expanded during the COVID-19 pandemic, these modes of data collection also predate the pandemic. These approaches may offer some important ways forward for innovative evaluations of VAWG programming during the pandemic. At the same time, it is important to carefully consider the strengths and limitations of such approaches. Understanding best practices and pitfalls of RDC is critical to continuing to advance research and programming for women and girls to ensure survivors are not left behind in the tide of the COVID-19 pandemic. For these reasons, a scoping review was conducted from February to March 2021 to map evidence related to RDC approaches as they pertain to evaluating interventions around VAWG. Scoping reviews are used to rapidly map the landscape of an emerging area of research/evidence for the purpose of understanding major themes and concepts, identifying gaps and opportunities, and guiding future research or systematic reviews, rather than conducting formal critical appraisal. ${ }^{18} 19$ This scoping review adheres to the Preferred Reporting Items for Systematic Reviews and Meta-Analyses (PRISMA) extension for scoping reviews reporting guidelines.

The purpose of this scoping review is to describe the state of peer-reviewed literature pertaining to evaluations of VAWG interventions that collect data remotely, across high-income and low-income settings. In addition to collating information on how remote evaluations are conducted, this scoping review centres on ethics and participant safety to identify best practices and lessons learnt for the purpose of guiding future evaluations of VAWG programming during COVID-19 and other emerging infectious disease outbreaks, epidemics and pandemics.

\section{METHODS}

This scoping review was conducted to answer the following questions:

1. What are commonly used modes of RDC employed for evaluating interventions aimed at preventing or responding to VAWG?

2. What are the primary concerns around ethics and safety when collecting sensitive data remotely and how can these concerns be mitigated?

3. What are best and good practices for using RDC to evaluate VAWG interventions?

\section{Eligibility criteria}

Inclusion and exclusion criteria were defined prior to beginning the search. Sources considered for inclusion consisted of peer-reviewed, empirical research that aimed to evaluate interventions as well as guidance papers on how to collect evaluation data remotely. No restrictions were placed on study setting; we considered sources from both high-income and low-income countries. Qualitative, quantitative, and mixed-methods evaluation research pertaining to an intervention-programme, tool, service, or policy-were included, and we did not restrict based on study design. Sources were eligible for inclusion according to whether they: (1) focused on evaluating an intervention; (2) employed RDC tools; and (3) measured women/girls' direct victimisation experiences or other VAW-related outcomes from women/girls, such as attitudes or help-seeking behaviour. Sources were 


\begin{tabular}{|c|c|c|}
\hline $\begin{array}{l}\text { Domain A } \\
\text { VAWG }\end{array}$ & $\begin{array}{l}\text { Domain B } \\
\text { Evaluation }\end{array}$ & $\begin{array}{l}\text { Domain } \mathrm{C} \\
\text { Remote data collection }\end{array}$ \\
\hline $\begin{array}{l}\text { Gender-based violence } \\
\text { Intimate partner violence } \\
\text { Rape } \\
\text { Battered women } \\
\text { Domestic violence } \\
\text { - Sex offences } \\
\text { GBV } \\
\text { Violence against women* } \\
\text { VAW } \\
\text { VAWG } \\
\text { V IPV } \\
\text { Sexual violence }\end{array}$ & $\begin{aligned} \text { - Evaluation study } \\
\text { Evaluat* } \\
\text { - Randomized controlled trial } \\
\text { - Epidemiologic studies } \\
\text { - Case-control studies } \\
\text { - Retrospective studies } \\
\text { - Cohort studies } \\
\text { - Follow-up studies } \\
\text { - Longitudinal studies } \\
\text { - National longitudinal study } \\
\text { - Adolescent health } \\
\text { - Prospective studies } \\
\text { - Controlled before-after studies } \\
\text { - Cross-sectional studies historically controlled } \\
\text { study }\end{aligned}$ & $\begin{array}{l}\text { Remote data } \\
\text { Telephone interview* } \\
\text { Telephone } \\
\text { Digital data collect* } \\
\text { Mobile app* } \\
\text { Computer assisted telephone } \\
\text { interview* } \\
\text { Computer assisted self interview* } \\
\text { Computer assisted personal } \\
\text { interview* } \\
\text { Computer assisted interview* } \\
\text { Online questionnaire* } \\
\text { Online survey* } \\
\text { Oideo interview* } \\
\text { Online data collection }\end{array}$ \\
\hline
\end{tabular}

Table 1 represents the Boolean search terms for keywords and Medical Subject Headings optimised for Ovid Medline, searched in February 2021.

excluded if they did not meet these criteria. Given the decline in landline telephones in high-income countries, the extremely low prevalence of this form of communication in low-income countries, and the tendency for landline telephones to be used in random digit dialling and nationally representative surveys as opposed to evaluations, data collection methods that relied exclusively on landline telephone interviews were also excluded. RDC of gender-based violence (GBV)-related outcomes among men and boys was outside of the scope of our research question and warrants a separate review of the literature. We also did not consider sources that were unavailable in English or conference abstracts.

\section{Search strategy}

First, using a Boolean search strategy we searched by title, abstract, keywords and Medical Subject Headings according to the following three domains: VAWG, evaluation and RDC (table 1). The research team collaborated to develop the search strategy, drawing on their collective expertise from previously published systematic reviews in areas related to VAWG $^{1720-22}$ and the search strategy was executed by $\mathrm{LV}$, who received previous training regarding systematic reviews. The database search was conducted in February of 2021 and optimised for Medline, Embase, Cochrane Central Register of Controlled Trials, Global Health APA PsycInfo and Scopus. No search limitations or filters were applied. Refer to online supplemental appendix A for the final search strategy, optimised for each database.

\section{Screening}

Following the initial database extraction and elimination of duplicate sources, the screening process took place in three stages: (1) title and abstract; (2) full text review; (3) extraction of included studies. The screening and extraction were completed using Covidence. The screening team, consisting of six reviewers, worked independently to evaluate the titles and abstracts and full texts of all publications that were identified through the search. IS and LV led a team training to explain the purpose of the scoping review and the eligibility criteria prior to the reviewers beginning the screening. The title and abstract of each study were simultaneously assessed to identify whether publications addressed the central components of this review and one vote determined whether the article forwards at each stage. Consultation and consensus with the wider research team occurred when the reviewers raised questions about a given source with respect to the eligibility criteria. Studies that met all criteria defined above were included in the full text review, where one vote moved the study forward to extraction.

To facilitate extraction, a data-charting form was developed by IS and LV to determine specific areas and themes to extract from the included publications. The data-charting fields were developed by consulting available sources pertaining to best practices on RDC during COVID- $19^{12}$ and in relation to our a priori thematic areas of interest: ethics and safety. IS, LV and SM pilot tested the utility of the data-charting form by randomly selecting two articles that were eligible for extraction and independently filling out the charting form for each article. The charting fields were further refined following this pilot test. Discrepancies were resolved via consensus and following the final version of the charting form, SM and LV completed the data extraction independently for the remaining articles eligible for extraction. The datacharting form was updated regularly with the addition of each additional article and reviewed by the research team for consistency. As this is a scoping review which is 
only evaluating existing literature, no patients or human subjects were involved in the design or execution of this study.

\section{Analysis}

The extracted data from each article were analysed according to the research questions. To better understand the types of remote evaluations being employed to examine VAWG interventions, we first catalogued general information on each study, including the authors, year of publication, country of data collection, name and goal of intervention, sample size and characteristics, minimum age of inclusion, mode of RDC employed, GBV-related outcome collected, study design, length of follow-up and the content provided to the control group, if any.

Characteristics of the RDC protocols were then synthesised and analysed across two primary domains: procedural ethics and safety. Specifically, studies were first reviewed based on their consideration of and adherence to standard procedural ethics, including those relating to the consenting process, establishment of referral pathways for those who disclose abuse or a need for other services during data collection, measures taken to ensure data security and ethical practices around compensation. For the purposes of this review, we have defined data security to include any study protocols that minimise the likelihood that someone other than the study team members or a respondent can see or access the respondent's data.

Next, included evaluations were reviewed for their safety-related eligibility criteria, considerations around study communication and scheduling, and safety considerations for participants during data collection. When conducting a study on VAWG, considerations around respondent safety should be made before, during and after data collection. As RDC may compromise certain aspects of safety germane to in-person studies, researchers conducting RDC need to build in additional mechanisms to protect participants' safety. Part of the onus of ensuring participant safety falls directly on the study team-relating to how the researchers schedule interviews and communicate with participants, for instance-and the remaining responsibility comprises due diligence in embedding certain safety mechanisms within data collection tools and carefully explaining additional steps respondents can take to protect themselves.

\section{Patient and public involvement}

As this study presents a scoping review of evaluation methods, involving patients was not applicable.

\section{RESULTS}

\section{Selection of sources of evidence}

In total, 1175 articles were identified and extracted from Medline, Embase, Cochrane Central Register of Controlled Trials, Global Health APA PsycInfo and Scopus. Three additional studies were located through discussions with VAWG practitioners and scholars. Once the 353 duplicates were remoted, 825 articles entered title and abstract screening; 22 were included in full text review and read by one team member to determine whether or not they were appropriate for extraction and charting. Based on the inclusion and exclusion criteria, information was extracted from 14 sources. Refer to figure 1 for the PRISMA flow diagram.

\section{Study characteristics}

As shown in table 2, the studies included in this review were notably limited in their geographical reach. A majority of the studies were conducted in North America (eight in the USA, two in Canada), two were implemented in Australia and two in Europe (one in Belgium and one in Sweden). Among the 14 studies included in this review, half (7) collected data using telephone interviews, 5 used online surveys and 2 used mobile applications. It is noteworthy that of the seven telephone-based evaluations, only one collected follow-up data in more than one time period; in contrast, two of the five online surveys and both app-based evaluations collected data at multiple follow-up periods. This finding may lend support to the notion that researchers including follow-up data in their studies identified apps or online surveys as more appropriate modes of data collection due to lower costs, time needs, ease of use or other reasons. It is also important to note that researchers can employ more than one type of RDC tool, thereby offering participants multiple options at follow-up. Greater flexibility in follow-up modes of data collection may result in higher response rates. The studies included in this review comprised 10 randomised controlled trials, 1 evaluation in which three treatments were compared for the same group of participants, 2 quasi-experimental trials and 1 exploratory study. RDC was used to gather information on a range of VAWG-related outcomes, including experiences of physical, psychological, and sexual violence, ${ }^{23-28}$ use of various safety-seeking behaviours and activities, ${ }^{24} 2629$ and survivor experiences of support services in clinical and social service settings. ${ }^{30} 31$

A majority of the included studies evaluated interventions intended to support survivors of VAWG (10 out of 14 studies), three studies assessed interventions aimed at preventing VAWG and the final study examined a bystander intervention programme. Evaluated interventions varied widely, including interactive online platforms that engaged women in exploring individual safety and health and identified personalised safety planning strategies, ${ }^{7}$ informational and activity booklets mailed to mother-adolescent pairs that encouraged discussion of GBV-related topics, ${ }^{23}$ and a texting intervention in which university students received daily texts for 30 days designed to prompt engagement in positive behaviour changes within their romantic relationships. ${ }^{25}$

\section{Procedural ethics}

Although all 14 studies collected data remotely, only 7 obtained consent without any in-person contact; the remaining studies obtained consent in person, ${ }^{28-30}$ 


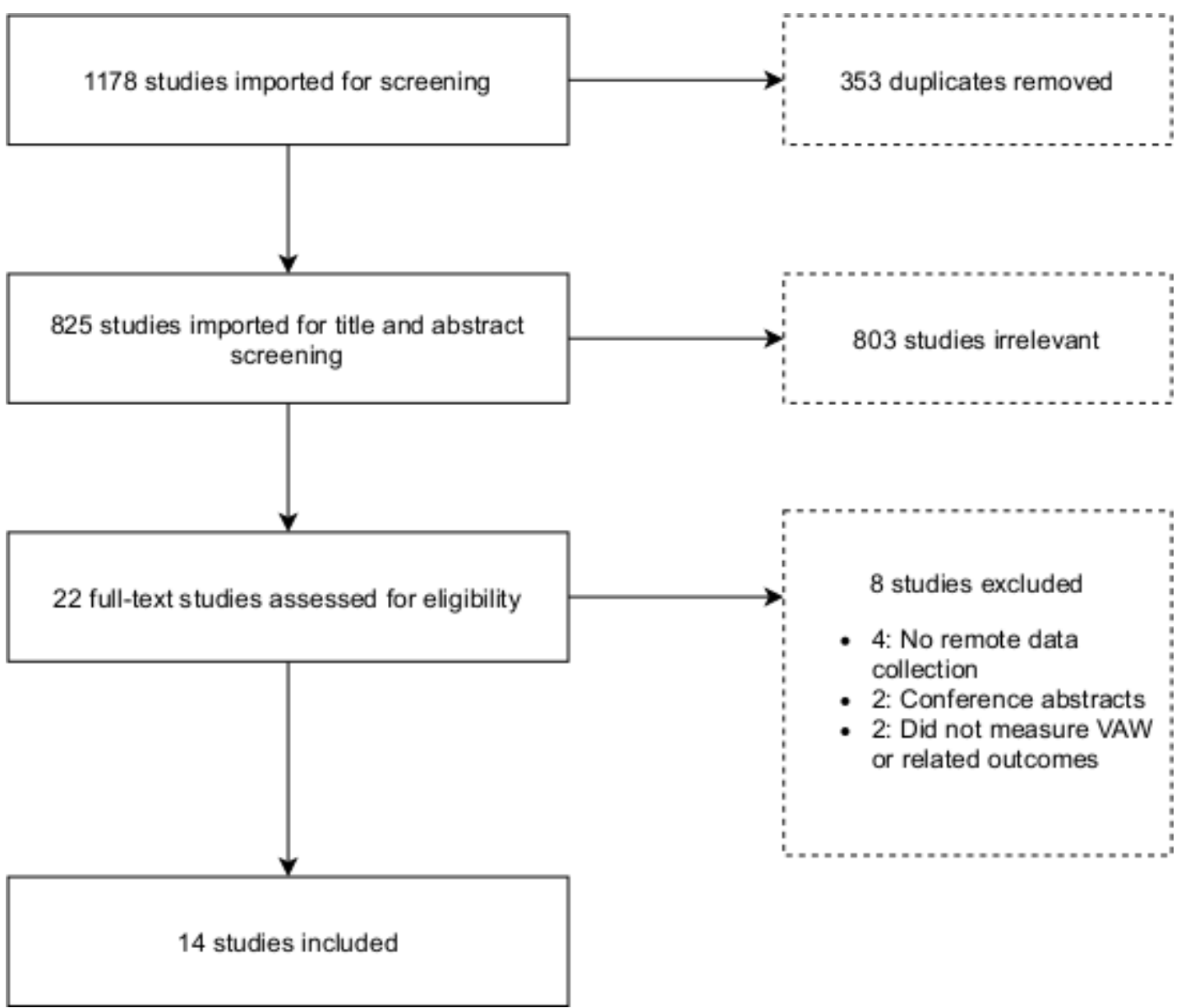

Figure 1 PRISMA diagram. PRISMA, Preferred Reporting Items for Systematic Reviews and Meta-Analyses; VAW, violence against women.

used a mix of in-person and remote procedures, ${ }^{72327}$ or did not mention how consent was collected ${ }^{25} 3132$ (see table 3). Remotely obtained consent was acquired using the mode that matched data collection; that is, studies that collected data using telephonic interviews obtained verbal consent over the phone and studies that employed online or app-based surveys acquired consent through an emailed consent form or by using a consent information sheet combined with participant acknowledgement of consent on the study website. To avoid exposing participants' identities on the data portal, one study procured a waiver of signed consent. ${ }^{24}$ Importantly, although one study noted the inclusion of women with disabilities in the study population, there was no mention of additional considerations taken to ethically obtain phone-based consent for these participants. ${ }^{30}$

Eight of the 14 included studies established referral pathways for respondents as per best practice protocol when collecting VAWG data. For example, Tarzia et $a l^{33}$ note the importance of identifying quality GBV services, such as domestic violence hotlines or psychosocial services, that study team members can provide to participants should they need support. In order to minimise contamination of the control group through the introduction of violence-related services, one study provided referrals for respondents in the intervention group only, an approach which directly counters ethical best practice. ${ }^{28}$ Finally, four studies did not mention a referral pathway $^{23253031}$ and the remaining study provided referrals within the intervention itself but not as part of data collection. $^{29}$

Only two of the seven telephone-based studies noted measures taken to ensure data security; Van Parys $e t a l^{28}$ randomly assigned case numbers to participants and a researcher outside the research team managed the study ID key, and Westwood et al, ${ }^{31}$ who did not collect any follow-up data, collected all phone-based data anonymously. Among studies employing app-based or webbased data collection activities, five required respondents to have a username and password, and one also used a dual-factor authentication approach. ${ }^{34}$ In the study by Senn and Forrest, ${ }^{35}$ participants were given a personal code on enrolment and Hatch et $a t^{25}$ made no mention of data security considerations.

Finally, there is a long history of debate and exploration over what is appropriate and acceptable compensation to incentivise participation in evaluations, as well as when incentivising crosses a line and becomes coercion. ${ }^{36}$ We looked at frequency and levels of compensation for remote evaluations to explore whether these are 


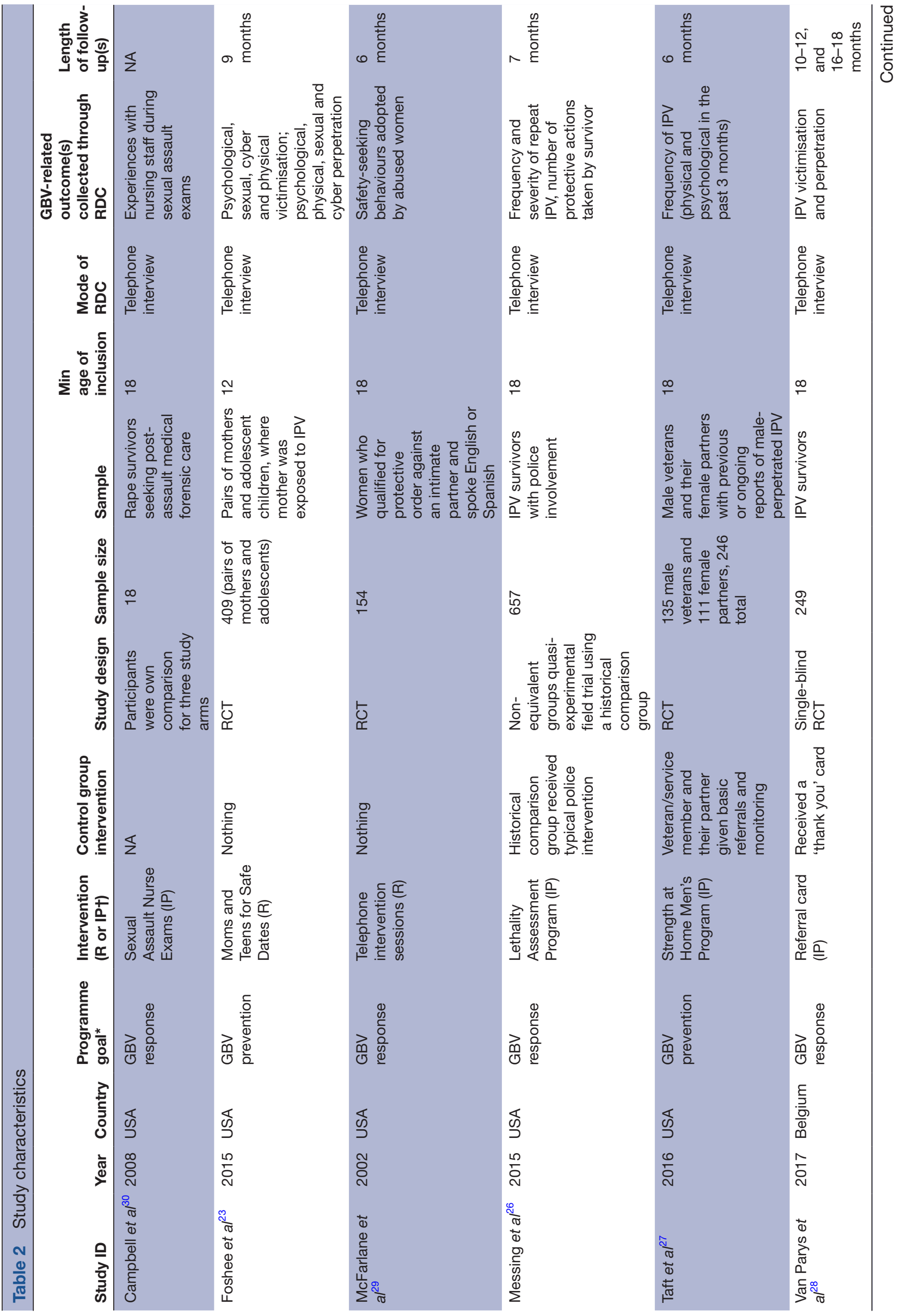

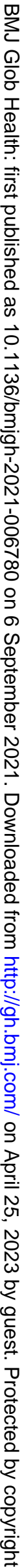




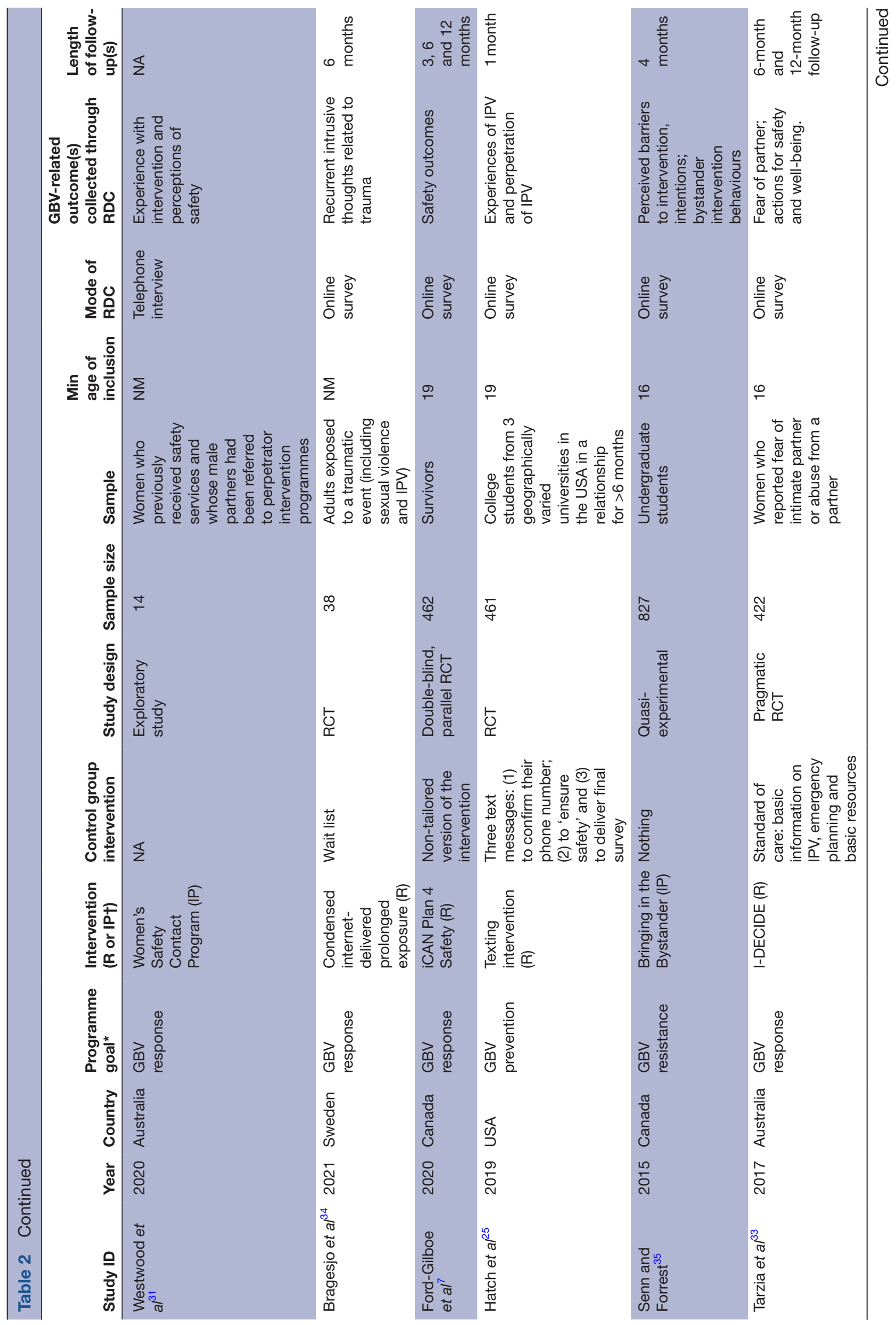




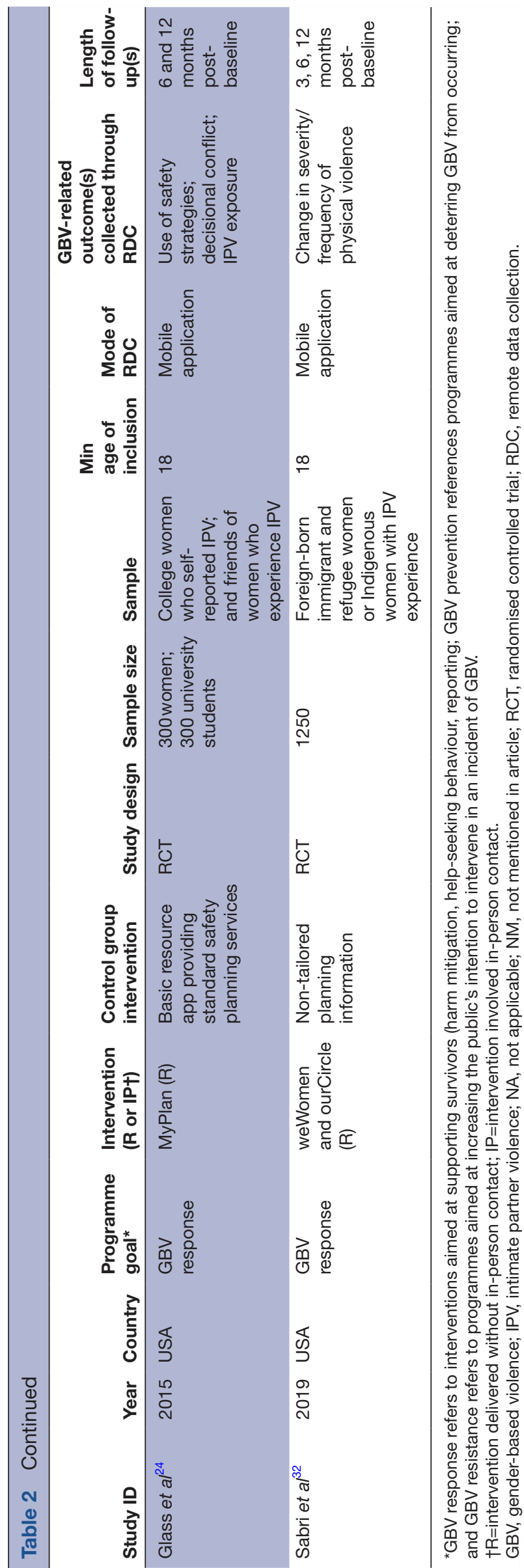

in line with current parameters around ethical compensation. Nine of the 14 included studies reported some use of compensation, with amounts ranging from $\$ 10$ to $\$ 50$ per round of data collection. As several studies included multiple rounds of follow-up, participants in a few evaluations were able to earn $\$ 100$ or more over the course of the evaluation. ${ }^{7243} 33$ Further, among studies that included multiple points of data collection, a graduated approach to compensation was employed, where each additional completed point of data collection resulted in greater compensation. ${ }^{724} 293235$ For example, Sabri et a $\hat{l}^{22}$ offered respondents $\$ 20, \$ 25, \$ 30$, and $\$ 40$ for completing data collection at baseline, 3 months, 6 months and 12 months, respectively. Compensation most commonly took the form of gift cards, sent either electronically or via mail.

In addition to offering compensation, several studies included automated and manual reminder messages to complete scheduled data collection in order to maximise retention. ${ }^{72432335}$ Reminder messages were facilitated through phone call, text, or email depending on the RDC tool used and the form of contact information collected from respondents at their point of entry into the evaluation study. Studies that collected information on one to two safe contacts per respondent were able to retain participation in the case of relocation and subsequent contact information change. ${ }^{33}$ Second, studies also reported sending multiple reminders to participants, with three to five maximum attempts being reported. ${ }^{24} 3335$ Thus, collecting diversified forms of secure contact information (ie, email, phone, friends/family contact information $)^{33}$ and contacting participants at regular intervals throughout the evaluation to confirm their contact information $^{24}$ are important considerations to maximise retention beyond compensation. Finally, among the seven studies that employed online surveys or mobile applications as RDC tools, four reported the inclusion of technological support, should participants require assistance. $^{7243233}$

\section{Safety}

Eight studies established explicit safety criteria for participating in the study, many of which related to the remote nature of data collection, such as being able to provide a safe phone number, safe email or having access to a safe computer. However, while studies used such criteria, further details on how researchers established that a participant's phone, email or computer was 'safe' were not provided. Additionally, one study noted that women who were known to still live with an abusive partner were not eligible to participate in order to reduce chances of a perpetrator overhearing or seeing data collection activities $^{23}$; however, it is important to recognise that this approach is not fail safe as some survivors may opt not to disclose recent incidents of intimate partner violence. Safety-related protocols for communication between the study team and respondents were also reviewed. Respondents in four of the seven telephone-based studies were 


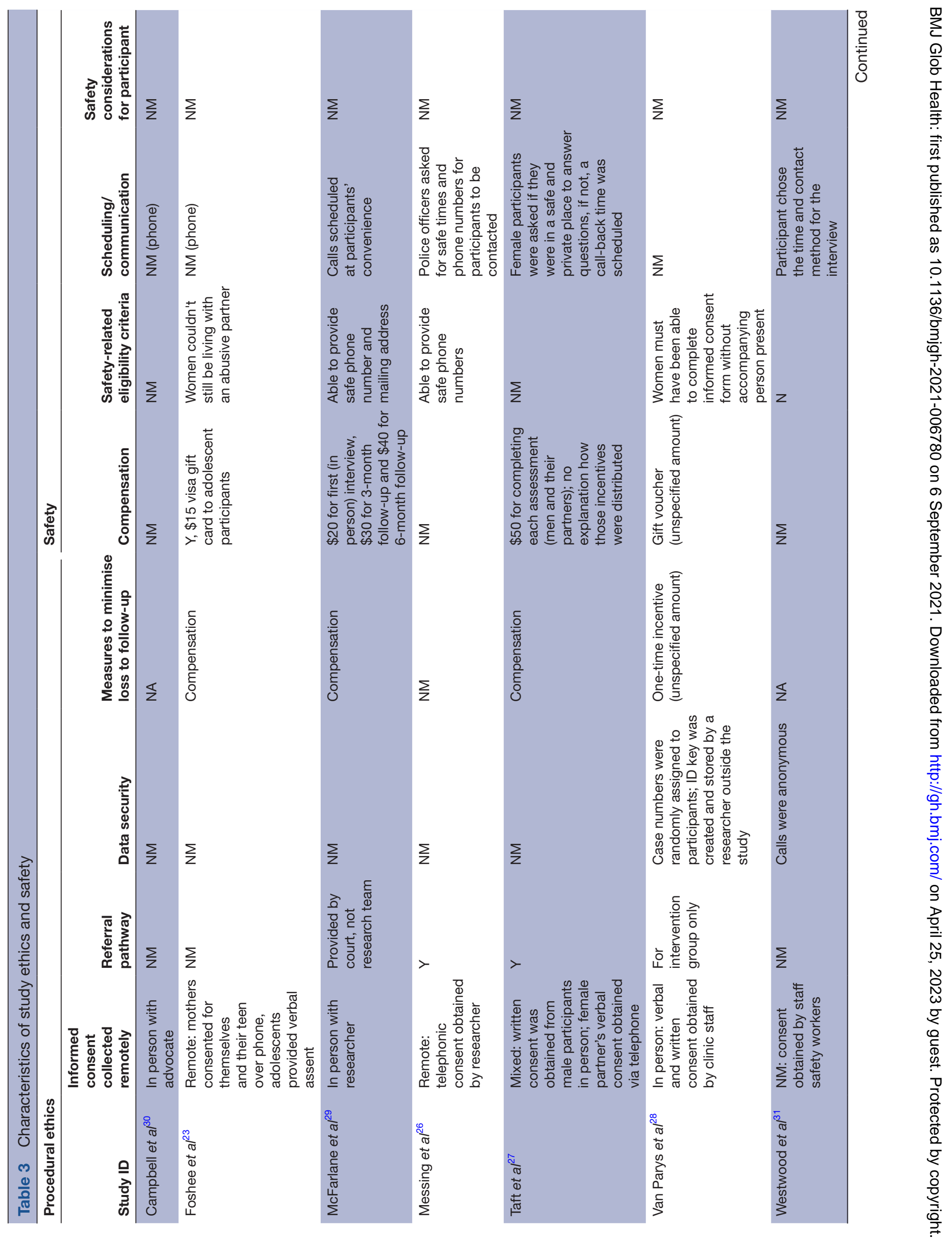




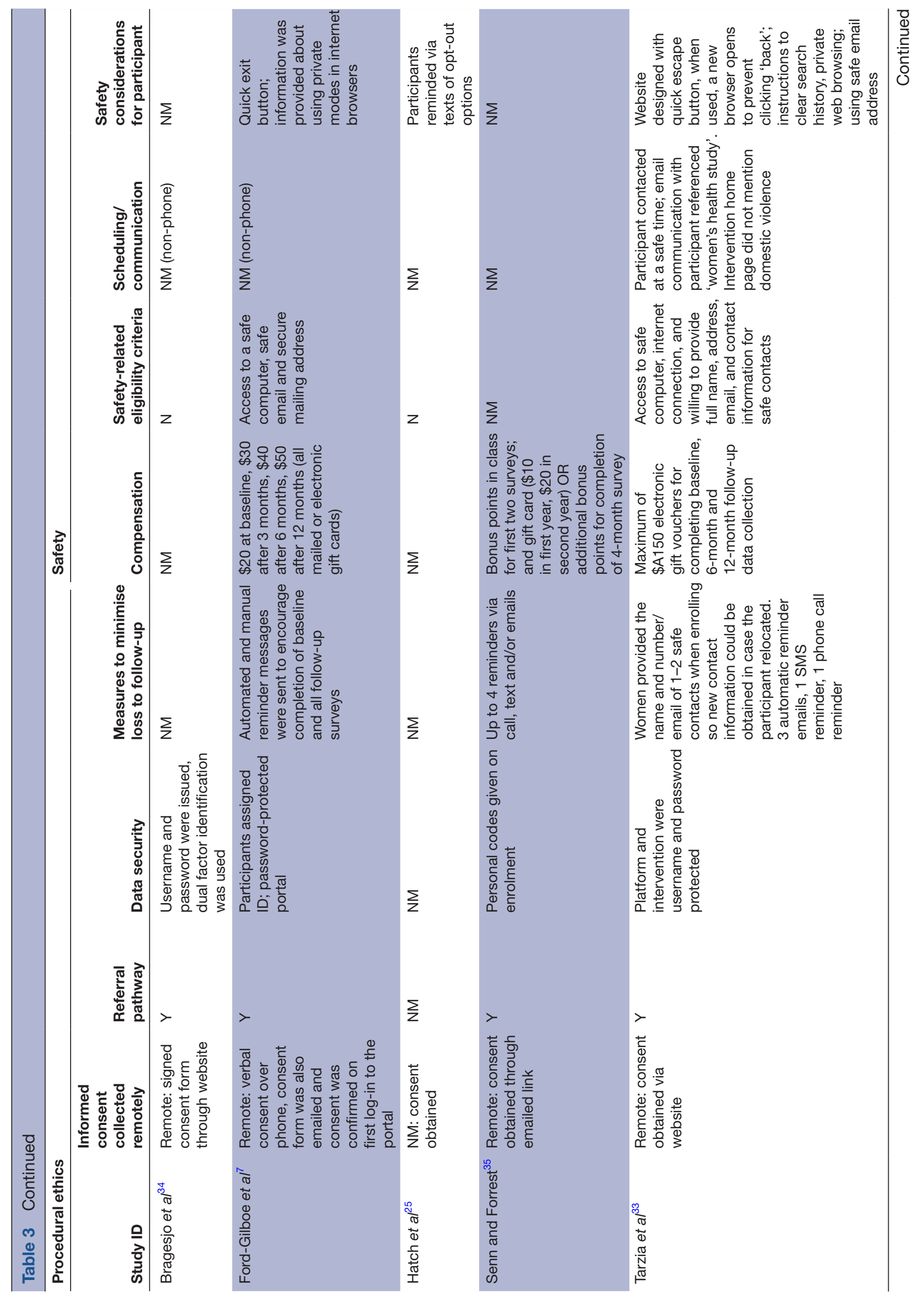




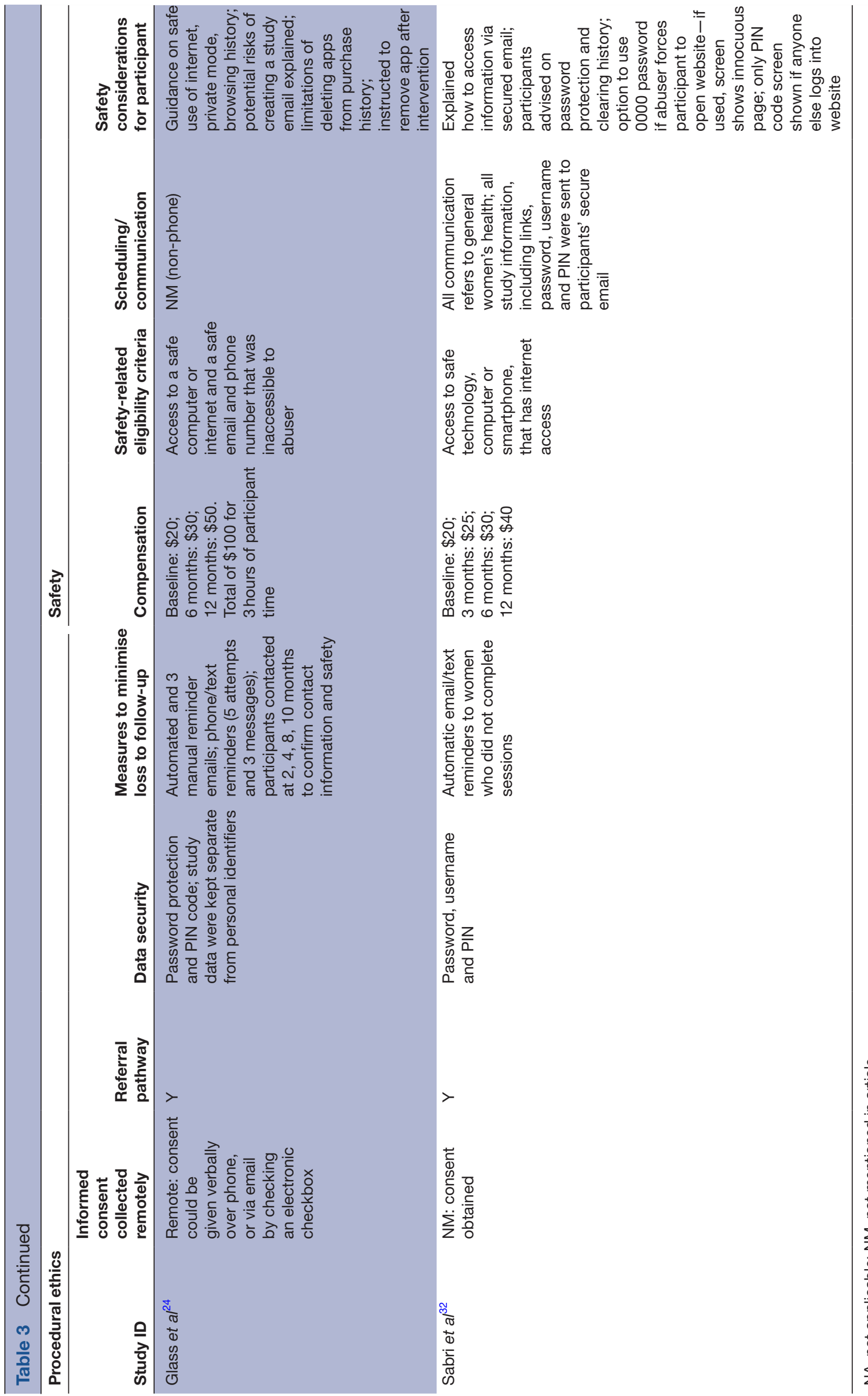


either asked for a safe time for the phone interview to take place or were asked by the researcher at the onset of the interview whether 'now was a safe time to talk', with calls rescheduled if necessary. ${ }^{26} 272931$ Among the seven non-telephone-based studies, for which study communications were often shared via email, two of the studies were referred to as ones on 'women's health' in order to mitigate participant risk. ${ }^{32} 33$

None of the studies employing telephonic interviews mentioned any additional steps taken by the research team to ensure safety for respondents beyond scheduling interviews at a time that was reportedly safe for the respondent. In contrast, a range of considerations were made for studies using web-based or app-based data collection. Four out of these seven studies provided respondents with guidance on using their browser in private mode and/or clearing their browsing history. ${ }^{72} 3233$ Additionally, Tarzia et a $l^{3}$ created a 'quick escape' button for the data collection website, allowing respondents to quickly open a new browser window on their screen; the survey website in Ford-Gilboe $e t a l$ s study also used a quick exit button. One study included an additional mechanism to protect respondents' safety and privacy: if a participant's abuser forced her to open the study website, the participant was able to enter ' 0000 ' on the login welcome page, prompting the opening of an innocuous website rather than the study page. ${ }^{32}$ Glass $e t a l,{ }^{24}$ who collected data using a phone application, also advised participants on how to delete the app after the study's completion in addition to explaining to participants the limitations of deleting apps. The remaining two web-based studies did not discuss any additional safety considerations for the data collection process.

\section{DISCUSSION}

The COVID-19 pandemic poses novel challenges to conducting VAWG intervention evaluation research. To better understand ethical and safety considerations, this scoping review maps evidence from peer-reviewed VAWG intervention evaluations conducted using RDC tools. Overall, findings from the review support the feasibility of conducting remote evaluations of VAWG interventions with some thoughtful planning, an encouraging finding for contexts where public health policies may limit movement and in-person interactions. Further, RDC tools can afford participants greater privacy and sense of control in how and when to report violence if, for example, they are able to access online or application-based surveys when it is most appropriate for them.

Research involving survivors and women and girls at risk of VAWG must address and mitigate threats to participant safety, namely risk of violence escalation. ${ }^{37}$ Additional safety considerations are required when employing RDC tools as there is less control and knowledge of the setting in which data collection is taking place. While some studies evidenced commitment to securing participant safety, other studies were found to be lacking due diligence in ensuring safety safeguards at various stages of data collection. A minority of studies demonstrated and reported careful thought and planning of ethical procedures, safety and retention regarding remote VAWG intervention evaluations, offering guidance for those aiming to conduct remote evaluations of VAWGrelated interventions in the future. Based on findings from this review and additional guidance on best practice around data security, ${ }^{38-40}$ we have developed a checklist that researchers can reference when designing a remote evaluation of a VAWG-related intervention (see figure 2). As remote evaluations of VAWG-related interventions become more widespread, Institutional Review Boards (IRBs) will need to ensure their review of potential studies takes into account the added nuances around safety and confidentiality when employing remote methods of data collection; figure 2 also highlights checklist items IRBs should look for during their review process.

For evaluations conducted during the COVID-19 pandemic, careful consideration and due diligence are especially critical for the first item on the checklist: establishing available and quality referrals for all study participants. Due to the substantial funding needs required to combat COVID-19, governments across the globe have been forced to deprioritise GBV services in order to redirect funding to COVID-19 containment. ${ }^{41}$ Further, stayat-home orders and fear of contracting COVID-19 have prevented some women from accessing services that are available. ${ }^{42}$ Researchers evaluating VAWG interventions may need to think creatively about ways to connect women to appropriate services, which in turn may benefit women and girls who are isolated and disconnected from sources of social support, even in the absence of COVID-19.

Although other more novel forms of RDC, such as video interviews through Zoom, Google Meet or other similar online platforms, and text message surveys (eg, using WhatsApp) did not present in this review, the COVID-19 pandemic has ushered in a proliferation of these methods for research within the public health sector and beyond. ${ }^{43}$ These approaches are likely to remain popular long after the pandemic and may warrant additional considerations around safety and ethics. At the same time, these modes of data collection-particularly those that make use of video-may help to foster rapport between the participant and researcher and thus potentially increase disclosure of GBV. Finally, we opted not to include approaches like Audio Computer-Assisted Self-Interview, as this method requires some on-the-ground presence, but such technology may also serve as a method of interest for RDC. ${ }^{4-46}$

It is important to note that none of the eligible studies in our search came from low/middle-income countries (LMICs) or humanitarian settings. There may be additional barriers to conducting remote evaluations in these settings. For example, studies conducted in these resource-limited settings may not have the budget to support the provision of phone data/credits or compensation for reimbursements to maximise retention. 


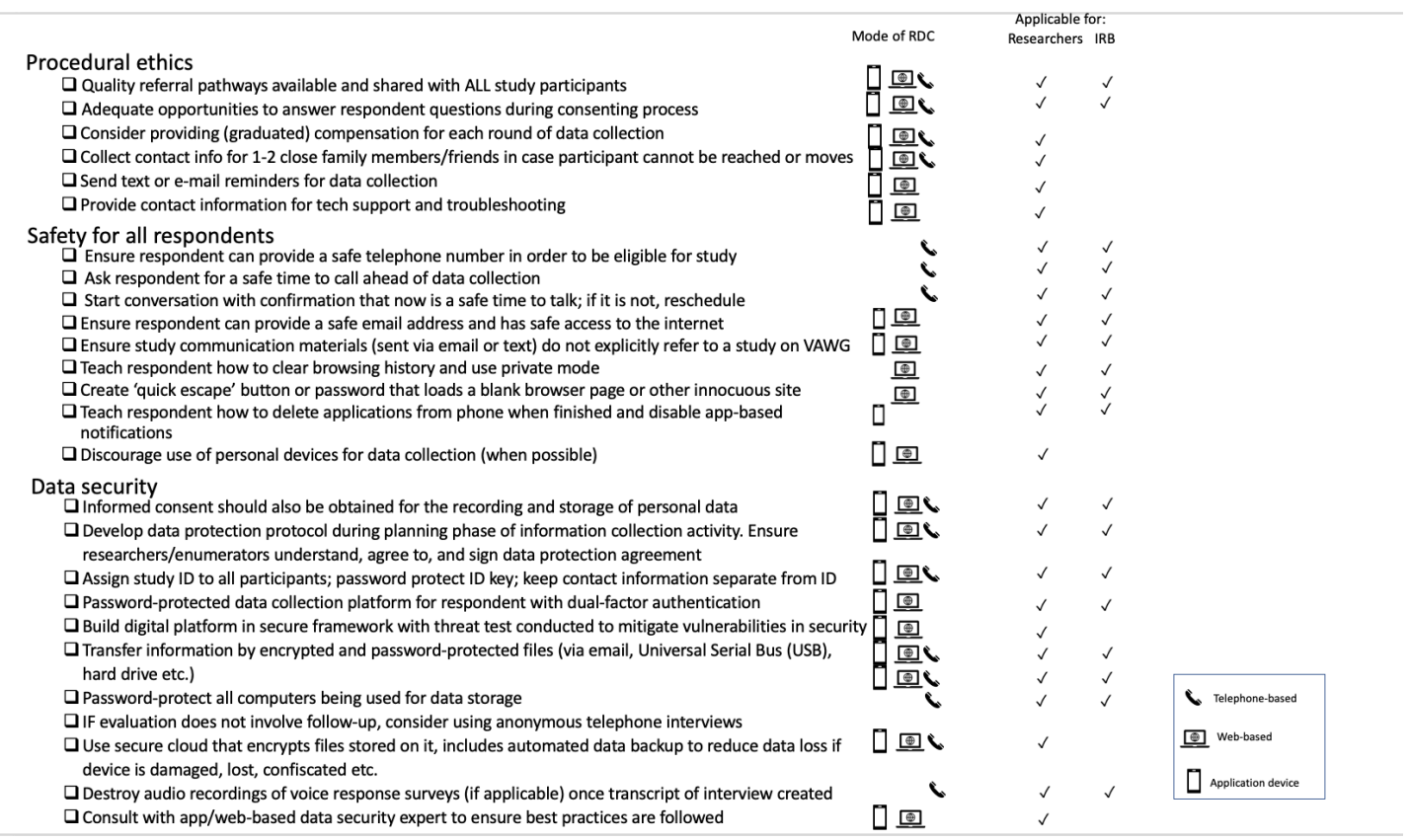

Figure 2 Remote evaluations of VAWG-related interventions: a guiding checklist for ensuring procedural ethics, respondent safety and sample retention. IRB, Institutional Review Board; RDC, remote data collection; VAWG, violence against women and girls.

Additionally, the general availability of access to smartphones, cellphones and internet may be limited in certain settings. Gendered attitudes towards women and girls' use of technology and the gender digital divide, where women have disproportionately reduced access to digital tools and digital literacy, are also important factors to consider. ${ }^{47} 48$ These issues pose concerns for ensuring all women and girls have access to technologybased VAWG interventions and are included in evaluation research. The best practice guidance distilled above may need to be further explored in these settings, where women and girls may not have access to these options. Further research can also help to identify strategies for promoting norms around women's and girl's access to and use of technology, in order to minimise disapproval or safety risks targeted toward female participants.

Other considerations for LMIC and humanitarian settings concern the availability and accessibility of referral services, participant literacy, maintaining privacy/confidentiality, and network constraints. ${ }^{4349}$ The COVID-19 pandemic has constrained VAWG services and so it is prudent for researchers to conduct a mapping of available referral services particularly in LMIC and humanitarian settings. ${ }^{49} 50$ Moreover, the completion of online surveys and use of mobile applications may be contingent on adequate literacy. It is important for researchers to offer participants a choice of RDC tools such as mobile phones or voice memos that would enable women and girls with low literacy to participate. Lastly, the inability to conduct face-to-face interviews in a private setting places the burden on participants to ensure their safety and confidentiality. ${ }^{43}$ This is of particular concern in humanitarian settings where persons face added stressors and insecurity. Lastly, the internet infrastructure and network capacity of a setting, irrespective of digital device ownership, may result in data upload and survey question dissemination delays. ${ }^{43}$

Also of note was the lack of studies that included younger populations. This observation may reveal a natural ethical recognition that it is more difficult to guarantee safety and confidentiality for younger populations when using RDC tools. For example, children's mobile devices may be monitored by caregivers, compromising the privacy and confidentiality of data shared through this platform. However, while RDC tools may usher in additional considerations around safety for children, efforts should be made to address these potential issues in order to support children's right to participate in research that involves them. ${ }^{51}$ Examples of the added risks and complexities researchers might need to consider when employing RDC tools with younger populations include designing tools that match the participants' levels of language and digital literacy, ensuring participants have devices that cannot be accessed by caregivers or others in the family (this may necessitate an in-person initial interaction with participants to provide such a device), and how to address issues around mandatory reporting laws in the study setting. A risk analysis should also be conducted prior to any data collection to ensure that the use of technology with children is culturally appropriate. ${ }^{52}$

Finally, we had originally also hoped to explore a third area in this review that distilled components related to methodological rigour and data quality. We know, for example, that phone interviews are likely to require a shorter administration time in order to minimise 
respondent fatigue and drop-off, ${ }^{53}$ and that more anonymous ways of disclosing violence (eg, through a selfadministered online survey) are likely to foster higher rates of disclosure than face-to-face interviews. ${ }^{53}{ }^{54}$ Ultimately, the kinds of details we were hoping to exploresuch as how scales or other measures were adapted for remote methods of data collection, whether the measures had been validated with the method employed, and whether any forms of bias specific to the mode of data collection were noted-were not provided in the articles, thus preventing us from conducting this analysis. Recognising the length limitations for many peer-reviewed publications, researchers might usefully consider including such methodological information in an online supplemental file 1 .

In terms of methodological implications, social desirability bias may be decreased when reporting experiences of violence in a private and confidential setting over web-based platforms. ${ }^{55}$ However, real or perceived confidentiality/privacy breaches may affect responses in that participants may feel surveilled by family members/ partners and answer in ways that would underestimate VAWG to avoid further violence or stigmatisation. Interactive voice response surveys, mobile apps or onlinebased surveys may also be used to mitigate interviewer fatigue and associated reductions in data quality. ${ }^{56}$ Further, to mitigate attrition, automated reminders for survey completion can be facilitated through SMS, mobile application push notifications or automated phone calls. Researchers employing RDC tools may have to adapt existing measurement scales or instruments; for example, researchers operating in settings where internet is unaffordable may have to condense onlinebased surveys or offer binary or ordinal response choices over SMS ${ }^{57}$ Measurement tools specifically designed to measure VAWG using RDC tools may need to be further developed and validated. Lastly, in the absence of randomisation and an appropriate control group, use of RDC tools to evaluate programmes could lead to selection biases given that eligibility criteria such as the ownership of a private device systematically exclude women and girls of lower socioeconomic status and could bias evaluation results.

Findings from this scoping review should be considered alongside a few limitations. First, due to the rapid nature of this review and resource limitations, the review could not accommodate two reviewers per source. As such, it is possible that articles were excluded that would have otherwise been deemed eligible by a second reviewer. Similarly, given the rapid expansion of studies during the COVID-19 pandemic, it is possible that additional eligible studies were published between the database extractions and publication of this review. However, we are confident that the range of protocols and practices employed in the included studies allowed for saturation of key themes and best practice implications for the design of remote evaluations in the field of VAWG. Third, it is important to acknowledge that studies may have incorporated additional study protocols related to our areas of interest, but simply did not report on these practices. Finally, none of the included studies reported adverse events or conducting any follow-up to understand how effective their data collection approaches were in keeping women safe; as such, our judgements and conclusions are mostly drawn from accepted best practice.

\section{CONCLUSION}

As a means of avoiding face-to-face interactions, the COVID-19 era has ushered in an expansion of RDC and programme evaluations. This scoping review presents an inventory of remote evaluations of interventions related to VAWG, examining study characteristics, ethical procedures and safety protocols. Drawing from our findings and known best practice for collecting data from survivors and on VAWG, we offer a practical checklist for researchers designing such remote evaluations in the future. In lieu of collecting data in person, where the researcher and participant can meet privately, researchers must ensure additional and creative protocols are implemented to protect the safety of participants. Although findings from this review speak to the feasibility of conducting remote evaluations of VAWG interventions in high-income settings, further research on additional considerations needed for LMIC and emergency contexts is warranted.

Twitter Luissa Vahedi @LuissaVahedi

Contributors IS and LS conceived of the study. IS, LV and SM created the inclusion/exclusion criteria, reviewed studies and extracted data for analysis. All authors contributed to writing and reviewing the manuscript.

Funding This study is made possible by the support of the American People through the US State Department Bureau of Population, Refugees and Migration.

Disclaimer The findings of this study are the sole responsibility of the contributing authors and do not necessarily reflect the views of the US Government.

Competing interests None declared.

Patient consent for publication Not required.

Provenance and peer review Not commissioned; externally peer reviewed.

Data availability statement Data sharing not applicable as no datasets generated and/or analysed for this study.

Open access This is an open access article distributed in accordance with the Creative Commons Attribution Non Commercial (CC BY-NC 4.0) license, which permits others to distribute, remix, adapt, build upon this work non-commercially, and license their derivative works on different terms, provided the original work is properly cited, appropriate credit is given, any changes made indicated, and the use is non-commercial. See: http://creativecommons.org/licenses/by-nc/4.0/.

\section{ORCID iD}

Lindsay Stark http://orcid.org/0000-0002-8775-9735

\section{REFERENCES}

1 UNICEF, UNFPA, UNODC, UNSD, UNWomen. Violence against women prevalence estimates, 2018. global, regional and national prevalence estimates for intimate partner violence against women and global and regional prevalence estimates for non-partner sexual violence against women. Geneva: World Health Organization, 2021.

2 García-Moreno C, Zimmerman C, Morris-Gehring A, et al. Addressing violence against women: a call to action. Lancet 2015;385:1685-95. 
3 Stark L, Seff I, Reis C. Gender-Based violence against adolescent girls in humanitarian settings: a review of the evidence. Lancet Child Adolesc Health 2021;5:210-22.

4 Tappis H, Freeman J, Glass N, et al. Effectiveness of interventions, programs and strategies for gender-based violence prevention in refugee populations: an integrative review. PLoS Curr 2016;8. doi:10.1371/currents.dis.3a465b66f9327676d61eb8120eaa5499. [Epub ahead of print: 19 Apr 2016].

5 UN Women. Violence Against Women and Girls Data Collection during COVID-19 [Internet]. 2020 Jun [cited 2021 Jan 11]. (UN Women Ending Violence Against Women (EVAW) COVID-19 Briefs vol. 2). Report No.: 2. Available: https://www.un-ilibrary.org/publichealth/violence-against-women-and-girls-data-collection-duringcovid-19_6c50a2a9-en

6 Decker MR, Wood SN, Kennedy SR, et al. Adapting the myPlan safety APP to respond to intimate partner violence for women in low and middle income country settings: APP tailoring and randomized controlled trial protocol. BMC Public Health 2020;20:808.

7 Ford-Gilboe M, Varcoe C, Scott-Storey K, et al. Longitudinal impacts of an online safety and health intervention for women experiencing intimate partner violence: randomized controlled trial. BMC Public Health 2020;20:1-17.

8 Hegarty K, Tarzia L, Valpied J, et al. An online healthy relationship tool and safety decision aid for women experiencing intimate partner violence (I-DECIDE): a randomised controlled trial. Lancet Public Health 2019;4:e301-10.

9 Ellsberg M, Heise L, Ellsberg L, et al. Researching violence against women: a practical guide for researchers and activists. Washington DC: WHO, PATH, 2005

10 World Health Organization. Putting women first: ethical and safety recommendations for research on domestic violence against women. World Health Organization, 2001.

11 Ebert JF, Huibers L, Christensen B, et al. Paper- or web-based questionnaire invitations as a method for data collection: crosssectional comparative study of differences in response rate, completeness of data, and financial cost. J Med Internet Res 2018:20:e24

12 Bhatia A, Peterman A, Guedes A. Remote data collection on violence against children during COVID-19: a Conversation with experts on research priorities, measurement and ethics (Part 2), 2020. Available: https://www.unicef-irc.org/article/2004-collectingremote-data-on-violence-against-children-during-covid-19-aconversation.html [Accessed 6 Dec 2020].

13 Peterman A, Bhatia A, Guedes A. Remote data collection on violence against women during COVID-19: a Conversation with experts on ethics, measurement \& research priorities (Part 1), 2020. https:// www.unicef-irc.org/article/1997-remote-data-collection-on-violenceagainst-women-during-covid-19-a-conversation-with.html

14 Davies SE, Bennett B. A gendered human rights analysis of Ebola and Zika: locating gender in global health emergencies. Int Aff 2016;92:1041-60

15 Onyango MA, Resnick K, Davis A. Chapter 8: Gender-Based Violence Among Adolescent Girls and Young Women: A Neglected Consequence of the West African Ebola Outbreak. In: Schwartz D, Abramowitz S AJ, eds. Pregnant in the time of Ebola, 2019: 121-32.

16 Peterman A, O'Donnell M. COVID-19 and violence against women and children: a third research round up for the 16 days of activism, 2020: 1-12.

17 Stark L, Meinhart M, Vahedi L, et al. The syndemic of COVID-19 and gender-based violence in humanitarian settings: Leveraging lessons from Ebola in the Democratic Republic of Congo. BMJ Glob Health 2020;5:e004194.

18 Armstrong R, Hall BJ, Doyle J, et al. 'Scoping the scope' of a cochrane review. J Public Health 2011;33:147-50.

19 Arksey H, O'Malley L. Scoping studies: towards a methodological framework. Int J Soc Res Methodol 2005;8:19-32.

20 Rubenstein BL, Lu LZN, MacFarlane M, et al. Predictors of interpersonal violence in the household in humanitarian settings: a systematic review. Trauma Violence Abuse 2020;21:31-44.

21 Stark L, Robinson MV, Seff I, et al. The effectiveness of women and girls safe spaces: a systematic review of evidence to address violence against women and girls in humanitarian contexts. Trauma Violence Abuse 2021:1524838021991306.

22 Stark L, Ager A. A systematic review of prevalence studies of gender-based violence in complex emergencies. Trauma Violence Abuse 2011;12:127-34.

23 Foshee VA, Dixon KS, Ennett ST, et al. The process of adapting a universal dating abuse prevention program to adolescents exposed to domestic violence. J Interpers Violence 2015;30:2151-73.

24 Glass N, Clough A, Case J, et al. A safety APP to respond to dating violence for College women and their friends: the MyPlan study randomized controlled trial protocol. BMC Public Health 2015; $15: 871$.

25 Hatch SG, Roddy MK, Doss BD, et al. Texts 4 romantic relationships - a randomized controlled trial. $J$ Couple Relatsh Ther 2020;19:115-35.

26 Messing JT, Campbell J, Webster DW, et al. The Oklahoma lethality assessment study: a quasi-experimental evaluation of the lethality assessment program. Soc Serv Rev 2015;89:499-530.

27 Taft CT, Macdonald A, Creech SK, et al. A randomized controlled clinical trial of the strength at home men's program for partner violence in military veterans. J Clin Psychiatry 2016;77:1168-75.

28 Van Parys A-S, Deschepper E, Roelens K, et al. The impact of a referral card-based intervention on intimate partner violence, psychosocial health, help-seeking and safety behaviour during pregnancy and postpartum: a randomized controlled trial. BMC Pregnancy Childbirth 2017;17:346.

29 McFarlane J, Malecha A, Gist J, et al. An intervention to increase safety behaviors of abused women: results of a randomized clinical trial. Nurs Res 2002;51:347-54.

30 Campbell R, Adams AE, Patterson D. Methodological challenges of collecting evaluation data from traumatized Clients/Consumers. Am J Eval 2008;29:369-81.

31 Westwood T, Wendt S, Seymour K. Women's Perceptions of Safety After Domestic Violence: Exploring Experiences of a Safety Contact Program. Affilia 2020;35:260-73.

32 Sabri B, Njie-Carr VPS, Messing JT, et al. The weWomen and ourCircle randomized controlled trial protocol: a web-based intervention for immigrant, refugee and Indigenous women with intimate partner violence experiences. Contemp Clin Trials 2019;76:79-84

33 Tarzia L, Valpied J, Koziol-McLain J, et al. Methodological and ethical challenges in a web-based randomized controlled trial of a domestic violence intervention. J Med Internet Res 2017;19:e7039.

34 Bragesjö M, Arnberg FK, Särnholm J, et al. Condensed internetdelivered prolonged exposure provided soon after trauma: a randomised pilot trial. Internet Interv 2021;23:100358.

35 Senn CY, Forrest A. "And then one night when I went to class..." The impact of sexual assault bystander intervention workshops incorporated in academic courses. Psychol Violence 2016;6:607-18.

36 Williams EP, Walter JK. When Does the Amount We Pay Research Participants Become "Undue Influence"? AMA J Ethics 2015;17:1116-21.

37 Anderson JC, Glass NE, Campbell JC. Conducting clinically based intimate partner violence research: safety protocol recommendations. Nurs Res 2017:66:405-9.

$38 \mathrm{Du} \mathrm{Z}, \mathrm{Xu}$ X, Wu Y. Case management, GBVIMS/GMVIMS+ and the COVID-19 pandemic, 2020: 14.

39 GBV AoR Helpdesk, UNICEF, GBV IMS, Plan International, UNFPA UNHCR, et al. COVID-19 guidance on remote GBV services focusing on Phone-based case management and Hotlines, 2021: 71.

40 World Health Organization. WHO ethical and safety recommendations for researching, documenting and monitoring sexual violence in emergencies. Geneva: WHO, 2007: 33.

41 John N, Roy C, Mwangi M, et al. COVID-19 and gender-based violence (GBV): hard-to-reach women and girls, services, and programmes in Kenya. Gend Dev 2021;29:55-71.

42 Viero A, Barbara G, Montisci M, et al. Violence against women in the Covid-19 pandemic: a review of the literature and a call for shared strategies to tackle health and social emergencies. Forensic Sci Int 2021;319:110650.

43 Hensen B, Mackworth-Young CRS, Simwinga M, et al. Remote data collection for public health research in a COVID-19 era: ethical implications, challenges and opportunities. Health Policy Plan 2021:36:360-8.

44 Falb K, Tanner S, Asghar K, et al. Implementation of AudioComputer assisted Self-Interview (ACASI) among adolescent girls in humanitarian settings: feasibility, acceptability, and lessons learned. Confl Health 2016;10:32

45 Rathod SD, Minnis AM, Subbiah K, et al. ACASI and face-to-face interviews yield inconsistent estimates of domestic violence among women in India: the Samata health study 2005-2009. J Interpers Violence 2011;26:2437-56.

46 Seff I, Steven S, Deitch J. Developing ACASI survey questionnaires in a conflict setting during the COVID-19 pandemic: using datainformed processes to address complexity, sensitivity, and functionality. under review.

47 Antonio A, Tuffley D. The gender digital divide in developing countries. Future Internet 2014;6:673-87.

48 Rowntree O, Bahia K, Butler C, et al. Connected women: the mobile gender gap report 2020. GSM Association, 2020. 
49 SVRI. SVRI knowledge exchange: pivoting to remote research on violence against women during COVID-19. Sexual Violence Research Initiative, 2020: 10. https://svri.org/svrinterest/svri-knowledgeexchange-pivoting-remote-research-violence-against-womenduring-covid-19

50 Roesch E, Amin A, Gupta J, et al. Violence against women during covid-19 pandemic restrictions. BMJ 2020;369:m1712.

51 United Nations. Convention on the rights of the child. General Comment No. 12: the right of the child to be heard. Geneva, Switzerland, 2009: 28. https://www2.ohchr.org/english/bodies/crc/ docs/AdvanceVersions/CRC-C-GC-12.pdf

52 Bennouna C, Mansourian H, Stark L. Ethical considerations for children's participation in data collection activities during humanitarian emergencies: a Delphi review. Confl Health 2017;11:5.

53 Barr AL, Knight L, França-Junior I, et al. Methods to increase reporting of childhood sexual abuse in surveys: the sensitivity and specificity of face-to-face interviews versus a sealed envelope method in Ugandan primary school children. BMC Int Health Hum Rights 2017;17:4.

54 Toktorbaeva N, Williams T, Abazbekova N, et al. Methodological lessons learned from conducting a population-based phone survey on nutrition practices in the Kyrgyz Republic. Curr Dev Nutr 2021;5:885

55 Crutzen R, Göritz AS. Social desirability and self-reported health risk behaviors in web-based research: three longitudinal studies. BMC Public Health 2010;10:720.

56 Elliott R. Interactive Voice Response vs Computer Assisted Telephone Interviewing for Survey Research [Internet]. GeoPoll, 2019. Available: https://www.geopoll.com/blog/interactive-voiceresponse-vs-computer-assisted-telephone-interviewing-research/ [Accessed 4 Aug 2021].

57 Ybarra ML, Agaba E, Chen E, et al. Iterative development of in this toGether, the first mHealth HIV prevention program for older adolescents in Uganda. AIDS Behav 2020;24:2355-68. 